[Agr. Biol. Chem., Vol. 31, No. 4, p. 448 451, 1967]

\title{
Immunoelectrophoretic Analysis of Wheat Flour Albumins
}

\author{
By Zen-ichiro Hamauzu, Daizo Yonezawa and Hiromu Shimomura* \\ Laboratory of Food Chemistry, College of Agricultue, \\ University of Osaka Prefecture, Sakai \\ * Mukogawa Women's University, Nishinomiya \\ Received October 29, 1966
}

\begin{abstract}
Albumin preparations from four kinds of wheat flour (Durum, Manitoba No. 2, Western White and Norin No. 26) were analyzed by immunoelectrophoresis. Seven to eleven components were detected for each preparation. They were classified and designated on the basis of the electrophoretic mobility $(R)$ and the character of precipitin lines formed by antigen-antibody reactions.
\end{abstract}

\section{INTRODUCTION}

Pence has investigated wheat albumins by paper electrophoresis and detected eleven components at maximum. ${ }^{1}$ However, resolution of patterns in the paper electrophoresis was not so good. Recently Grabar and Williams ${ }^{2,3)}$ originated immunoelectrophoresis which has been frequently used for the study of serum albumins and many other protein systems. Since this method is excellent in resolution and characterization of component proteins, we tried to apply it to the study of wheat albumins. Under proper conditions, good resolution was obtained and the number of components detected was similar to that reported by Pence. ${ }^{11}$ Four kinds of wheat flours including Durum were used as materials.

\section{MATERIALS AND METHODS}

Materials

Albumins. Albumins were prepared according to the method of Pence and Elder.4) Four kinds of wheat flours, Durum (Canada), Manitoba No. 2 (Canada), Western White (U.S.A.) and Norin No. 26 (Japan) were used as materials. The flours were ex-

1) J.W. Pence, Cereal Chem., 30, 328 (1953).

2) P. Grabar and C. A. Williams, Biochim. Bicphys, Acta, 10, 193 (1953).

3) P. Grabar and C. A. Williams, ibid., 17, 67 (1955)

4) J. W. Pence and A. H. Elder. Cereal Chem., 30, 275 (1953) perimentally milled to $60 \%$ extraction at Nisshin Milling Flour Co., Kobe.

Immune serum. Wheat protein used for immunization was prepared as follows. Proteins were extracted with $1 \mathrm{M} \mathrm{NaCl}$ from a mixture of above mentioned four kinds of wheat flour, and precipitated from the extract by the addition of solid ammonium sulfate to $70 \%$ saturation. The precipitates were lyophilized after removal of salts by dialysis. To make an antigen-adjuvand emulsion, $2 \mathrm{~g}$ of the protein obtained above was dissolved in $20 \mathrm{ml}$ of $0.15 \mathrm{M}$ $\mathrm{NaCl}$ solution and insoluble parts were removed by centrifugation at $20,000 \times \mathrm{g}$. The supernatant solution was mixed with an adjuvand* in a ratio of one to one. Two rabbits were immunized. The antigenadjuvand emulsion $(1.2 \mathrm{ml})$ was injected subcutaneously into each rabbit. After eight weeks, the animals were injected again intramuscularly with $1 \mathrm{ml}$ of a freshly prepared emulsion. Bleeding was made two weeks after the second stimulation. The antiserum was preserved in a refrigerator with an addition of merthiolate (sodium ethylmercuric thiosalicylate, $0.01 \%)$.

Agar for electrophoresis, Agar purchased from Wako Pure Chemical Co. was used after deionization procedure as described by Crowle. $\left.{ }^{5}\right)$ This procedure is necessary to prevent the formation of precipitate with the phosphate buffer used and a large electroosmotic flow in the electrophoresis run.

* Freund complete adjuvand: A mixture of Arlacel A $1.5 \mathrm{ml}$, dried heat-killed tubercle bacillus $50 \mathrm{mg}$, and liquid paraffin $8.5 \mathrm{ml}$.

5) A.J. Crowle, "Immunodiffusion", Academic Press, 1961, p. 196. 


\section{Methods of immunoelectrophoresis}

Agar solution, $8.5 \mathrm{ml}$ of $1.25 \%$, in a phosphate buffer ( $\mathrm{pH} 7.6$, ionic strength 0.1) was poured on a $7 \times 12 \mathrm{~cm}$ plate glass. After agar was solidified, four wells ( $1 \mathrm{~mm}$ in diameter) were cut in a line perpendicular to the current application (see Fig. 1). Well

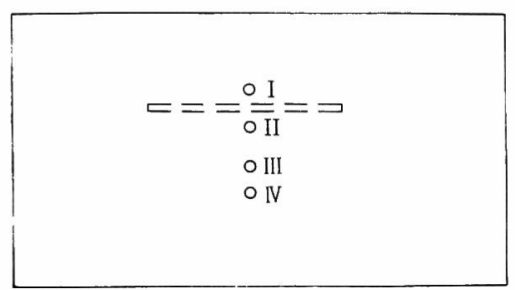

FIG. 1. Agar Gel Plate Used for Immunoelectrophoresis.

I: Well for sample protein; II: for Durum albumin; III: for BPB-stained bovine serum albumin (BPBBSA); IV: for o-nitroaniline (ONA). After the run agar was cut off along broken line and antiserum was placed in the slot.

I was fild with albumin samples to be analyzed and Well II with Durum albumin as a reference. Wells III and IV were filled with a solution of bovine serum albumins stained with bromphenolblue (BPB-BSA) and a saturated solution of 0 -nitroaniline in $40 \%$ methanol (ONA), respectively. BPB-BSA was used as an indicator to follow an extent of electrophoretic migration of the run, and ONA, visible with yellow colour, was an indicator of electro-osmotic flow, having practically on net charge at the range of $\mathrm{pH} 7.0$ to 8.8.6) Electrophoresis was carried out at a potential gradient of 6.8 to 7.0 volts per $\mathrm{cm}$ for thirty minutes in a cold chamber. After the run, a slot ( $5 \mathrm{~cm}$ in length; $1 \mathrm{~mm}$ in width) was cut in the middle of holes I and II along the protein migration, and antiserum was placed in it. The plate was allowed to stand for twenty-four hours in a tightly covered and humidified vessel at room temperature for development of antigen-antibody precipitation. Precipitin lines were photographed under an appropriate lighting without staining.

\section{RESULTS AND DISCUSSION}

Electrophoretic patterns of four kinds of wheat albumin used in this study are shown

6) H. Kikkawa and Z. Ogita, Kagaku, 18, 2 (1963). in Figs. 2 5. At least 7 to 11 components were detected from the patterns; 11 for Manitoba No. 2, 7 for Western White and Norin No. 26, and 8 for Durum. By paper electrophoresis, Pence ${ }^{11}$ also detected 11 components with the albumin preparation prepared in the same way as we did. However, it should be pointed out that Pence's components do not necessarily correspond to ours as judged from the mobility distribution described later (Fig. 6, see also Fig. 1 in reference (1)).

By immunoelectrophoresis, Grabar et al. ${ }^{71}$ detected 8 to 10 components with $0.5 \mathrm{~m} \mathrm{NaCl}$ soluble protein of wheat flour. However,
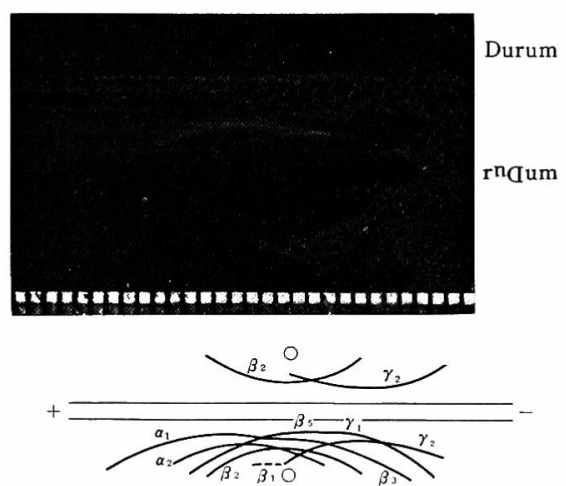

FIG. 2. Immunoelectrophoretic Pattern of Durum Wheat Albumin.
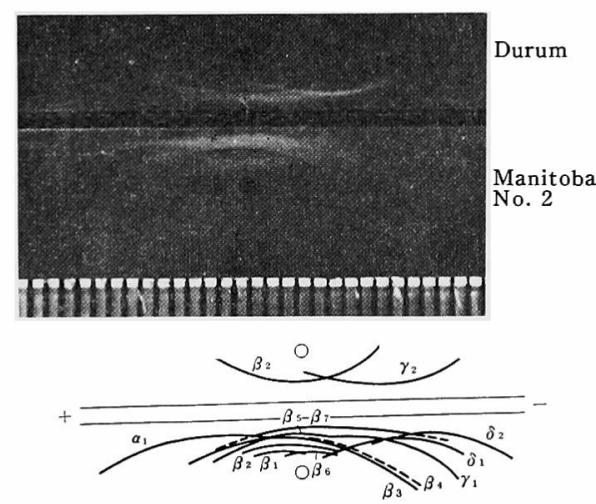

FIG. 3. Immunoelectrophoretic Pattern of Manitoba No. 2 Wheat Albumin with Durum Albumin as a Reference. 

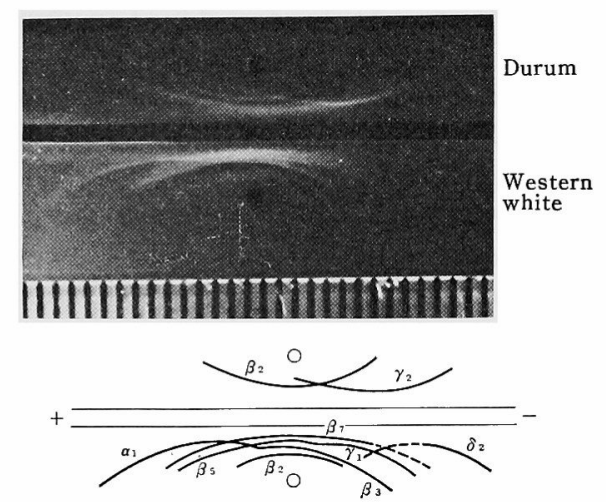

FIG. 4. Immunoelectrophoretic Pattern of Western White Wheat Albumin with Durum Albumin as a Reference.
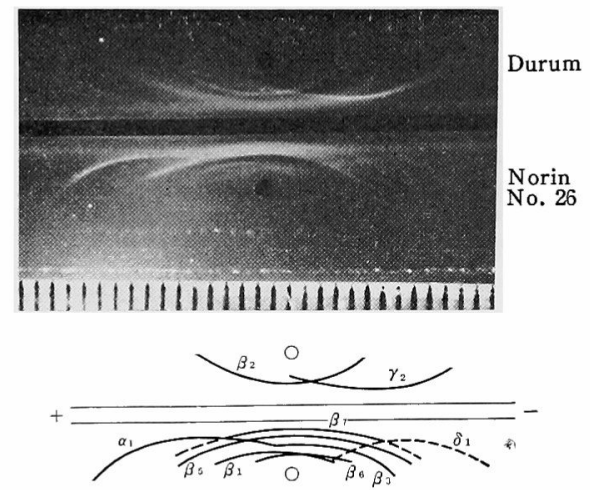

FIG. 5. Immunoelectrophoretic Pattern of Norin No. 26 Wheat Albumin with Durum Albumin as a Reference.

with wheat albumin they found much less components, though the exact number was not reported. They pointed out that prolonged dialysis against water caused reduction of the number of components and that 11 components reported by Pence could have been attributed to incomplete removal of globulins by dialysis.

Grabar et al. used a veronal buffer in their experiments. In our experience with a veronal buffer ( $\mathrm{pH} 7.9$ and ionic strength 0.05

7) P. Grabar, N. Benhamou and J. Daussant, Arch. Biochem. Biophys., Suppl. 1, 187 (1962).
0.1 , precipitin lines were flattened and consequently components with well-defined lines were reduced. This may be one reason for a decreased number of components detected by Grabar et al.

Though electrophoresis runs were made under the same conditions as possible, small deviation of the electrophoretic migration distance was inevitable from run to run. Therefore, to determine the electrophoretic mobility, a ratio, $R=M_{x} / M_{s}$, was adopted, where $M_{x}$ was the distance of electrophoretic migration for the component protein of wheat albumin and $M_{s}$ was that for the standard BPB-BSA migrated on the same plate. The distance of the electrophoretic migration was determined from the position of the protein after a run with the calibration for the electro-osmotic flow estimated from the shift of ONA spot. The position of the component protein of wheat albumin was determined from the precipitin line as a point on arc nearest to the slot of antiserum. $R$ values thus obtained are summerized in Table I and Fig. 6. In Fig. 6 , the center of the circle represents a mean value of $R$ in four repeated runs, and individual values are within the circle.

As shown in Fig. 6, the distribution of components was essentially the same in all the four kinds of wheat used, though con-

TABle I. Designations AND Mobilities $(R)$ OF ALbUMin Components of WheAT FlouR

$\begin{array}{lcccc} & \begin{array}{c}\text { Manitoba } \\ \text { No. 2 } \\ (R)\end{array} & \begin{array}{c}\text { Western } \\ \text { White } \\ (R)\end{array} & \begin{array}{c}\text { Norin } \\ \text { No. 26 } \\ (R)\end{array} & \begin{array}{c}\text { Durum } \\ (R)\end{array} \\ \alpha_{1} & 0.77 & 0.79 & 0.80 & 0.74 \\ \alpha_{2} & & & & 0.61 \\ \beta_{1} & 0.53 & & 0.52 & 0.50 \\ \beta_{2} & 0.48 & 0.43 & & 0.46 \\ \beta_{3} & 0.48 & 0.48 & 0.41 & 0.41 \\ \beta_{4} & 0.45 & & & \\ \beta_{5} & 0.44 & 0.43 & 0.42 & 0.38 \\ \beta_{6} & 0.43 & & 0.37 & \\ \beta_{7} & 0.38 & 0.44 & 0.42 & \\ \gamma_{1} & 0.25 & 0.20 & & 0.18 \\ \gamma_{2} & & & & 0.05 \\ \delta_{1} & -0.09 & & -0.05 & \\ \delta_{2} & -0.20 & -0.19 & & \end{array}$




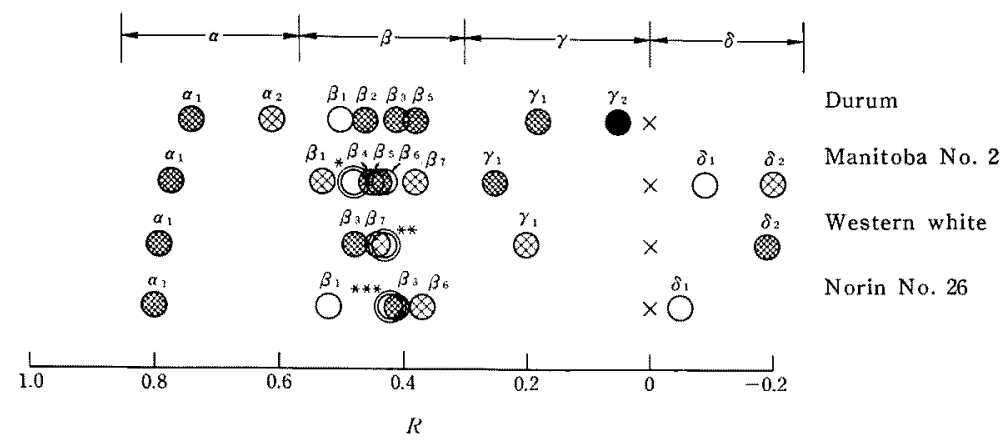

FIG. 6. Summarized Results of Electrophoresis with Four Kinds of Wheat Albumin.

The position of the circle represents the electrophoretic mobility $(R)$ obtained as a ratio of the electrophoretic migration distance between the individual component and BPB-stained bovine serum albumin. The mark in the circle represents the strength of the precipitin line $(>>>)$. The spot marked with asterisked concentric circles indicates that two components occupy the same spot; the two components are $\beta_{3} \beta_{2}, \beta_{3} \beta_{5}$ and $\beta_{5} \beta_{7}$ for the spot with one, two and three asterisks, respectively.

siderable variations were observed in Durum. Components were arbitrarily classified into four groups $\alpha, \beta, \gamma$ and $\delta$ according to their electrophoretic mobilities as shown in Fig. 6. Components were crowded in $\beta$-region, while only one or two were included in the other regions. It should be noted that $\delta$-group migrated to the cathod, indicating that the component proteins were positively charged at this $\mathrm{pH}$.

A precipitin line formed by the fusion of two arcs shows the presence of two components which are immunologically identical and electrophoretically different. Two components in $\beta$-group were characterized by this phenomenon.

Designation for individual components was made as shown in Table I. Corresponding components in different kinds of wheats were determined on the basis of the electrophoretic mobility and characters of the precipitin line such as strength, position and fusion of arcs. Thus $\beta_{3}$ and $\beta_{5}$ were characterized by the fusion of arcs with $\alpha_{1}$ and $\gamma_{1}$ respectively.

The electrophoretic pattern of Norin No. 26 appeared to lack in the characteristic fusion line of $\beta_{5}+\gamma_{1}$. This may be not due to the lack of these components but to their incomplete resolution. While Manitoba No. 2 contained both $\delta_{1}$ and $\delta_{2}$, Norin No. 26 lacked $\delta_{2}$ and Western White $\delta_{1}$. Durum showed a little different behavior in comparison with the other three. Component $\gamma_{2}$, which lacked in the other three formed a very sharp and deep precipitin line. Component $\alpha_{2}$ was also characteristic of Durum. On the other hand Durum lacked in the components belonging to $\delta$-group.

The results described above indicated that immunoelectrophoresis is a useful tool for the analysis of wheat albumins. Electrophoresis run no longer than thirty minutes gave a good resolution and the characterization of components was unique.

Acknowledgement. The authors are grateful to Dr. Z. Ogita of Osaka University for his kind advices for the technique of agar-gel electrophoresis, to Dr. T. Hirano and Dr. I. Yanagiya of University of Osaka Prefecture for helping us to prepare antigen and antiserum, to Dr. I. Hlynka of Grain Research Laboratory in Canada, the Osaka Agricultural Research Center and Nisshin Milling Flour Co. for presenting us wheat samples. 